\title{
Il trattamento di pazienti con sepsi grave mediante drotrecogin alfa: una valutazione economica con riferimento all'Italia
}

\author{
ANALISI \\ ECONOMICA
}

Carlo Lucioni*, Silvio Mazzi*, Chinn Christopher ${ }^{\circ}$

\begin{abstract}
Sepsis can be defined as a spectrum of clinical conditions caused by the immune response of a host to infection or trauma and characterized by systemic inflammation and coagulation. Particularly in elderly, immunocompromised and critically ill patients, sepsis is a major cause of morbidity and mortality in intensive care units (ICUs) worldwide. In the US, sepsis is the leading cause of death in noncoronary ICU patients. Drotrecogin alfa, or recombinant human activated protein $\mathrm{C}$, has antithrombotic, antiinflammatory, and profibrinolytic properties. Recently in a phase III trial (PROWESS), Drotrecogin alfa demonstrated significantly reduced mortality in severe sepsis patients at 28 days. In this trial important value factors for the assessment of costs and outcomes of severe sepsis were also considered.

The purpose of the present study is to determine the economic burden of the treatment with Drotrecogin alfa, according to a cost-effectiveness analysis based on the data of the PROWESS trial. The study has been adapted to the italian health environment.

As regards to the costs per surviving patient and costs per QALY (Quality Adjusted Life Year), the predicted cost-effectiveness ratio of drotrecogin alfa in severe sepsis patients is much lower than the standard values considered as acceptable in the international litterature.
\end{abstract}

Farmeconomia e percorsi terapeutici 2002; 3 (3): 171 - 177

\section{INTRODUZIONE}

La sepsi è, per la maggioranza dei pazienti, un disordine sistemico associato a un sottostante disturbo di tipo infiammatorio. Si tratta di una condizione patologica per la cui diagnosi non esistono ancora dei test adeguati e per il cui trattamento non si dà ancora una terapia standard efficace. La sepsi è la causa di morte più frequente nelle Unità di Terapia Intensiva (UTI) degli Stati Uniti, dove sono stati stimati circa 100.000 decessi per sepsi ogni anno [1].

Il drotrecogin alfa (Xigris ${ }^{\circledR}$ ), una versione ricombinante della proteina $\mathrm{C}$ attivata (dotrecogin alfa), si è dimostrato efficace nel ridurre la mortalità tra $i$ pazienti con sepsi grave ${ }^{i}$ [2]. Dato questo positivo risultato clinico, il presente lavoro si propone di valutarne la convenienza economica.

\section{MATERIALIEMETODI}

In ogni valutazione economica si richiede fondamentalmente che vengano messi in rapporto l'uso delle risorse (i costi sostenuti) con le relative conseguenze (i benefici conseguiti) per ciascuna delle alternative poste a confronto - che in questo caso sono solo due: trattare con dotrecogin alfa o no.

Ciò può essere fatto scegliendo opportunamente, secondo la situazione, fra alcune tecniche di analisi da tempo disponibili [3]. In quella adottata per questo lavoro, nota come analisi di costo/efficacia, i benefici (gli "effetti”, da cui il nome) sono misurati in termini fisici: casi diagnosticati, casi di malattia evitati, vite salvate, anni di vita guadagnati e così via (riferimenti che corrispondono a vari endpoint della letteratura clinica). In questo modo diventa possibile calcolare per ciascuna alternativa un

\section{* ADIS International/ Health Economics, \\ Milano \\ ${ }^{\circ}$ Ely Lilly $U K$}

\section{Nota $\mathbf{i}$}

Per una possibile

definizione di quest'ultima vedasi Bone [17] 
Nota ii

Questo indicatore economico prende il nome di rapporto costolefficacia. indicatore $^{\text {ii }}$ (costo per caso diagnosticato, per paziente guarito, per anno di vita guadagnato, a seconda dei casi) che può essere confrontato con quelli delle altre; si potrà così dire, ad esempio, che guadagnare un anno di vita costa meno con questa invece che con quell'alternativa e quindi avere un criterio oggettivo di preferenza e di scelta.

Da questa tecnica è derivata l'analisi di costo/utilità, che si caratterizza per fare riferimento a un unico criterio di misurazione dei benefici. Essa parte prendendo in considerazione l'anno di vita guadagnato (mutuandolo dall'analisi di costo/efficacia) apportandovi, però, un perfezionamento. Poiché, infatti, un anno di vita non è la stessa cosa per tutti (una guarigione, ad esempio, può preludere a una sopravvivenza in condizioni di salute più $o$ meno favorevoli), questa tecnica si propone di "pesare" quell'unità di misura in funzione della qualità della vita che effettivamente vi corrisponde - ovvero, ricorrendo a un termine tipico dell'economia, del grado di "utilità" percepita dal soggetto che la vive [4]. Si arriva così a una nuova unità di misura, nota, nella letteratura anglosassone, col nome di QALY (Quality Adjusted Life Year). Nonostante le evidenti difficoltà, metodologiche e operative, che questa tecnica comporta, la preferenza per l'adozione (quando possibile) del costo per QALY come indicatore di convenienza economica si va sempre più diffondendo e generalizzando.

Il metodo di valutazione qui adottato è, come premesso, quello dell' analisi di costo/efficacia (costo per paziente sopravvissuto e costo per anno di vita guadagnato) e di costo/utilità (costo per QALY). È stata seguita un'analisi di tipo incrementale, nel senso che i costi che entrano nei suddetti rapporti sono dati dalla differenza tra quelli sostenuti se si tratta con dotrecogin alfa e quelli incorsi senza alcun trattamento; e analogamente dicasi per $i$ rispettivi risultati in termini di efficacia (pazienti sopravvissuti, ecc.).

I consumi di risorse sanitarie che sono all'origine dei costi considerati nell'analisi sono quelli rilevati in uno studio clinico internazionale, di cui si dirà tra breve. Tuttavia, nell'intento di portare l'analisi stessa il più vicino possibile al contesto sanitario italiano, i costi unitari adottati per valorizzare quei consumi sono quelli vigenti o stimati in tale contesto.

A questo proposito è però necessaria una precisazione per quanto riguarda le degenze ospedaliere. Queste erano infatti state misurate nel suddetto studio in termini di giornate trascorse dal paziente rispettivamente in Unità di Terapia Intensiva (UTI) e in corsia (le differenze, in particolare sotto il profilo economico, fra pazienti in trattamento e no sono appunto legate anche alle diverse durate di degenza). Di conseguenza la presente analisi si trova orientata a procedere valorizzando con dei costi giornalieri le degenze ospedaliere rilevate; ma, per quanto tali costi siano stimati con riferimento all'Italia, così facendo essa non è congruente con la prassi, qui ormai in vigore da anni, del pagamento prospettico (DRG), che notoriamente prescinde, salvo eccezioni, dalla durata della degenza stessa.

$\mathrm{Ne}$ deriva che, per quanto riguarda il costo delle risorse ospedaliere, il punto di vista dell'analisi non è quello del Servizio Sanitario Nazionale ma piuttosto quello dell'ospedale che, in questo caso, può anche coincidere con quello della collettività come soggetto che sostiene in definitiva i costi sanitari reali.

Questo punto di vista, peraltro, si addice nel complesso anche al resto dei costi considerati nella presente analisi.

\section{La base clinica}

Il presente lavoro fa riferimento a uno studio internazionale (PROWESS), controllato con placebo, multicentrico, in doppio cieco, randomizzato, con la partecipazione di 1.690 pazienti distribuiti fra Stati Uniti, Canada, Australia, Nuova Zelanda, Sud Africa, Brasile e cinque Paesi europei: Belgio, Francia, Germania, Olanda, Spagna [2].

Lo studio si proponeva di accertare se il trattamento con dotrecogin alfa fosse in grado di ridurre la mortalità fra i pazienti con sepsi grave. I pazienti arruolati erano affetti da infiammazione sistemica e disfunzione organica da infezione acuta; dovevano ricevere un'infusione i.v. continua o di placebo $(\mathrm{N}=840) \mathrm{o}$ di dotrecogin alfa al dosaggio di $24 \mu \mathrm{g} / \mathrm{kg}$ di peso corporeo/ora $(\mathrm{N}=850)$, per un ciclo di 96 ore. L'endpoint primario era la morte per qualsiasi causa a 28 giorni dall' inizio dell'infusione.

Il tasso di mortalità risultò essere del 30,1\% nel gruppo placebo e del 24,1\% nel gruppo trattato. Sulla base dell' endpoint primario il trattamento con dotrecogin alfa fu dunque associato a una riduzione assoluta del rischio del 6,0\% (p $=0,005)$.

Per altro i pazienti con 2 o più disfunzioni d'organo alla baseline (quindi con una base di gravità superiore a quella media dei pazienti arruolati) facevano registrare a 28 giorni i seguenti tassi di mortalità:

$$
\begin{array}{ll}
\text { placebo: } & 33,3 \%(\mathrm{~N}=212) \\
\text { dotrecogin alfa: } & 26,9 \%(\mathrm{~N}=165) \\
\begin{array}{l}
\text { riduzione assoluta } \\
\text { del rischio: }
\end{array} & 7,3 \%(\mathrm{p}=0,126)
\end{array}
$$

In quest'ultimo sottoinsieme, dato il numero di pazienti relativamente ristretto, la dif- 
ferenza tra le mortalità non risulta statisticamente significativa $a^{\text {iii }}$ - potrebbe cioè essere accolta solo come indicazione di tendenza. Tuttavia, il dato di riduzione del rischio del 7,3\% viene adottato nel caso base della presente analisi - invece di quello generale $(6,0 \%)$ perché riferito a una popolazione che rispecchia l'indicazione europea approvata dalla EMEA.

Comunque, il dato generale del $6,0 \%$ viene ripreso nell'analisi di sensibilità.

Nel corso dello studio PROWESS, in parallelo alla rilevazione dei dati clinici sono stati sistematicamente raccolti anche dati sui consumi delle risorse sanitarie da parte dei pazienti. In particolare sono stati qui considerati i consumi dei pazienti con due o più disfunzioni d'organo. Sono questi i dati fondamentali che, insieme ad altri, entrano nel modello farmacoeconomico qui utilizzato per l'analisi.

\section{Il modello}

Si tratta di un' applicazione programmata in Excel (MS) che - molto schematicamente sviluppa i seguenti passi:

1) sulla base dei giorni trascorsi in UTI e in corsia dai pazienti trattati, valorizzati coi rispettivi costi per giornata (per questi ultimi, vedasi oltre), viene calcolato un costo complessivo medio di degenza per paziente trattato; analogamente si procede per il paziente non trattato (placebo). La differenza (il primo dei due costi è logicamente maggiore, poiché relativo a un uso più prolungato delle risorse nel corso dei 28 giorni osservati, data la minore mortalità) costituisce il costo ospedaliero incrementale per paziente trattato.

2) A questo viene aggiunto il costo del farmaco di trattamento (dotrecogin alfa). Dalle rilevazioni di PROWESS risulta che sono stati mediamente somministrati (quindi tenendo anche conto dei decessi repentini a inizio periodo) 31,2 vials da $5 \mathrm{mg}$ per paziente ${ }^{\mathrm{iv}}$.

3) Per avvicinare l'analisi alla realtà locale, il modello tiene conto che in Italia si impiega spesso, nel trattamento dei pazienti settici, l'antitrombina III (AT III). Il costo corrispondente a un dosaggio medio di $7.392 \mathrm{U}$ [5] viene dunque aggiunto a quelli del paziente non trattato (ciò che equivarrebbe a sottrarlo da quelli del paziente trattato), pesato per la sua frequenza d'impiego stimata del 56\% [6].

4) $\mathrm{Si}$ ottiene così il costo incrementale totale per paziente trattato.

5) Ancora per spingere la localizzazione dell'analisi, nel calcolo degli anni di vita me- diamente guadagnati il modello utilizza le tavole della vita attesa per anno di età e sesso della popolazione italiana [7], applicandovi i differenziali di mortalità - cioè i guadagni in sopravvivenza tra pazienti trattati e non (pure essi segmentati per età e sesso e pesati col numero dei sopravvissuti) - rilevati dallo studio PROWESS. Peraltro, al fine di tenere conto anche della particolare mortalità post-dimissione nei sopravvissuti a sepsi grave (mortalità legata a malattie concomitanti, o agli effetti della stessa sepsi o della prolungata degenza in UTI), viene applicato un coefficiente generale di aggiustamento pari a 0,51 [8]. Gli anni di vita guadagnati non sono scontati nel caso base mentre lo sono, al tasso del 3\%, nell' analisi di sensibilità.

6) Le stesse variabili che possono influire sulla mortalità dei sopravvissuti alla sepsi, possono anche inficiarne la qualità della vita. Questa può, in generale, essere misurata scegliendo fra diversi strumenti, che procedono analizzando e valutando il quadro clinico di un paziente; ciò che conta, ai fini di una valutazione economica, è arrivare a un dato sintetico che esprima il grado di utilità attribuibile a un determinato stato di salute, ovvero alla qualità della vita che gli è correlata. Ad esempio, una delle metodologie più impiegate in questo campo, denominata EQ-5D (EuroQol/Quality of Life in Europe - 5 Dimensions), attribuisce ad ogni situazione individuale osservata un coefficiente compreso tra 1 (in corrispondenza a una situazione di perfetta salute) e 0 (equivalente al decesso) [9]. Poiché nello studio PROWESS non erano stati raccolti dati in materia, il modello adotta un coefficiente di utilità stimato per pazienti settici a 180 giorni dopo la dimissione pari a 0,69 [10], come peso medio da attribuire a tutti gli anni di vita guadagnati.

\section{I costi unitari}

Costo di una giornata in UTI. Il valore adottato è tratto da un articolo di recente pubblicazione [11]. In tale studio esso è stato stimato su un campione finale di 6 UTI nel Nord Italia e 3 nel Centro ed è comprensivo dei seguenti costi:

Costi variabili: disposable (materiale per intubazione e assistenza respiratoria, cateteri, materiale per terapia infusionale), farmaci (emoderivati, antimicrobici, sedativi, soluzioni varie, preparati per nutrizione enterale, altri farmaci), sangue (ed emoderivati non compresi tra i farmaci), esami (ematochimici, microbiologici, diagnostica per immagini), fisioterapia.

\section{Nota iii}

Cioè non risponde al criterio standard di $p \leq 0,05$.

\section{Notaiv}

L'intero quantitativo necessario a trattare un paziente di $72,5 \mathrm{~kg}$ $(0,024 \mu \mathrm{g}$ di farmaco per $\mathrm{kg}$ di peso all'ora per 96 ore) sarebbe di circa 33,4 vials 


\section{Tabella 1}

Analisi di costo/ efficacia di dotrecogin alfa in Italia. Caso base

\begin{tabular}{|c|c|c|}
\hline \multicolumn{3}{|c|}{ Euro } \\
\hline Costo medio ospedaliero per paziente trattato & 20.912 & A \\
\hline Costo medio ospedaliero per paziente non trattato & 18.831 & B \\
\hline Costo ospedaliero incrementale per paziente trattato & 2.080 & $\mathrm{C}=\mathrm{A}-\mathrm{B}$ \\
\hline Costo del farmaco di trattamento (PCA) & 8.157 & $\mathrm{D}$ \\
\hline Costo dell'AT III & -1.579 & E \\
\hline Costo totale incrementale per paziente trattato & 8.658 & $\mathrm{~F}=\mathrm{C}+\mathrm{D}+\mathrm{E}$ \\
\hline Riduzione assoluta del rischio di mortalità & $7,3 \%$ & $\mathrm{G}$ \\
\hline Costo per paziente sopravvissuto & 119.329 & $\mathrm{H}=\mathrm{F}: \mathrm{G}$ \\
\hline Anni di vita guadagnati per paziente & 12,35 & I \\
\hline Costo per anno di vita guadagnato & 9.660 & $\mathrm{~J}=\mathrm{H}: \mathrm{I}$ \\
\hline Qualità della vita & 0,69 & $\mathrm{~K}$ \\
\hline Costo per QALY & 13.999 & $\mathrm{~L}=\mathrm{J}: \mathrm{K}$ \\
\hline
\end{tabular}

In generale, nello studio citato i dati sono stati ricavati dalla contabilità analitica per centri di costo. Gli esami sono stati monetizzati sulla base delle tariffe riportate nel Nomenclatore Tariffario. Per il costo del sangue ed emoderivati si è utilizzato il prezzo unitario di cessione tra servizi sanitari.

Costi fissi: personale sanitario laureato (anestesista-rianimatore), personale sanitario non laureato (capo sala, infermieri professionali), personale tecnico (ausiliario socio-sanitario), attrezzature.

I costi di personale sono stati calcolati, nello studio citato, individuando il numero degli addetti all'attività di reparto e moltiplicando la percentuale dell'orario lavorativo di ciascuno di loro dedicata alla gestione dei pazienti per il costo medio annuo del rispettivo profilo professionale.

Le attrezzature sono state valorizzate sulla base del costo e dell'anno di acquisto (e ipotizzando un periodo di ammortamento pari a 8 anni, a quote costanti, come prescritto dal DPR 917/86) o, in alternativa, del canone di locazione. In mancanza del costo originario, si è fatto riferimento ai valori correnti di mercato.

Costi di struttura: utenze, forza motrice, riscaldamento, manutenzione, pulizia, lavanderia, mensa, smaltimento rifiuti, spese amministrative.

La quota-parte annua di tali costi è stata imputata, sempre nello studio citato, col metodo dell' allocazione diretta a basi di ripartizione multiple (giornate di degenza erogate; metri quadri).

Nel complesso, i costi variabili assommavano al $28 \%$ del totale, i costi fissi al $65 \%$, i costi di struttura al $7 \%$.

In definitiva, il costo medio per giornata di degenza in UTI, riferito al 1995 nello studio citato e nel presente rivalutato al 2000 con l'indice del valore della lira [7], ammonta a euro $1.033,43$.

Costo di una giornata in corsia. Anche in questo caso, si tratta di un dato pubblicato [12]. Il procedimento con cui esso è stato stimato parte dalle tariffe (DRG) pagate agli ospedali dal SSN.

Sulla base della documentazione statistica pubblicata dal Servizio Sistema Informativo della Regione Lombardia, relativa a tutti i ricoveri ospedalieri (circa 1,5 milioni di casi) effettuati dagli ospedali pubblici regionali nel 1997, suddivisi per reparto, la stima del costo di una giornata di degenza in un dato reparto è avvenuta facendo riferimento:

- al costo medio generale di un singolo caso (calcolato dividendo il valore globale dei DRG pagati indistintamente a tutti gli ospedali regionali nel corso dell'esercizio per il numero totale dei casi);

al punteggio medio (in termini di utilizzo relativo delle risorse, che può essere superiore o inferiore all' unità) del reparto in questione; moltiplicando per tale punteggio il suddetto costo medio generale si viene implicitamente a tenere conto del mix di DRG del reparto stesso;

- alla degenza media registrata in tutti i reparti di quel tipo; dividendo il prodotto di cui al punto precedente per il numero di giorni di tale degenza si è ottenuto così un costo per giornata in reparto.

Questo dato è poi stato rivalutato al 2000. Con questo procedimento sono stati stimati i costi die dei tre reparti (medicina, chirurgia, traumatologia) dove risultavano tipicamente ricoverati i pazienti con sepsi dello studio clinico di cui sopra; i tre dati sono infine stati 
mediati con pesi proporzionali alla distribuzione dei pazienti stessi nei rispettivi reparti, ottenendo un dato finale di euro 299,54.

Farmaco di trattamento (dotrecogin alfa). Si è ipotizzato di adottare un prezzo (a ricavo industria) per confezione (vial) da $5 \mathrm{mg}$ di euro 261,25 (comprensivo dell'IVAal 10\%).

AT III. Il prezzo al pubblico è di euro 381,46 per $1.000 \mathrm{U}$ [13]. Trattandosi di un plasmaderivato, non si applica lo sconto Ospedali del $50 \%$.

\section{RISULTATI}

In linea col modello sopra descritto, la tabella 1 presenta i risultati dell' analisi del caso base.

Trattare i pazienti settici con dotrecogin alfa significa ridurre il rischio di mortalità, ma anche andare incontro a maggiori costi.

In Italia infatti un paziente settico trattato con dotrecogin alfa costa, in più di quanto già si spende per uno non trattato, mediamente euro 8.658 - per la massima parte $(94 \%$ circa) dovuti al costo del farmaco stesso. Per ogni paziente sopravvissuto grazie alla riduzione del rischio impiegando dotrecogin alfa, si incorre in un costo di euro 119.329 .

In media, il paziente sopravvissuto può attendersi ancora 12,35 anni di vita; ognuno di questi anni costa dunque euro 9.660. Tenendo però conto che, per quanto si è in precedenza osservato, la qualità della vita in tali anni non sarà piena $(100 \%)$ bensì stimabile attorno al $69 \%$, il costo per QALY risulta pari a euro 13.999 .

\section{ANALISIDISENSIBILITÀ}

Con questo supplemento d'indagine ci si propone di verificare se i risultati del caso base cambierebbero sostanzialmente (oppure no) qualora alcune delle assunzioni utilizzate per il lavoro venissero modificate. La casistica esaminata prevede tre nuove situazioni, con diversi esiti.

1) Riduzione del rischio di mortalità. Il dato generale, a livello di tutto lo studio
PROWESS, è pari al 6,0\%, contro il 7,3\% relativo ai pazienti con due o più disfunzioni. Quest'ultimo dato, che era stato adottato nel caso base, viene sostituito nell' analisi di sensibilità dal dato generale; una riduzione del rischio pari al $6,0 \%$, essendo inferiore alla precedente, è logicamente destinata a peggiorare tutti i parametri di costo efficacia valutati nel caso base.

2) Sconto degli anni di vita guadagnati. Mentre nel caso base questi non erano scontati, nell'analisi di sensibilità essi lo sono, al tasso (attualmente spesso applicato in letteratura) del 3\%; con questo procedimento, non tutti gli anni contano per intero (cioè per uno), bensì nel loro succedersi contano sempre meno di uno. Ciò significa attribuire un'importanza economica decrescente agli anni di vita guadagnati quando questi sono più lontani nel tempo. Anche in questo caso, i parametri del costo per anno di vita guadagnato e del costo per QALY non possono che peggiorare rispetto al caso base.

3) Dose di AT III. Secondo dati pubblicati [14], la dose di antitrombina somministrata in Italia al paziente settico sarebbe di 24.000 U (contro le 7.392 che sono state contemplate nel caso base). Poiché il costo dell' antitrombina è a carico del trattamento senza dotrecogin alfa, un aumento del costo stesso va a favore della cost effectiveness di dotrecogin alfa.

Nella tabella 2 sono riportati i parametri di costo/efficacia calcolati per le tre suddette verifiche di sensibilità, preceduti - per comodità di confronto - da quelli del caso base.

\section{DISCUSSIONE}

Per quanto riguarda la generalizzabilità degli esiti clinici attestati nelle sperimentazioni, non sembrano esserci problemi. Per quanto riguarda invece le informazioni sull'uso delle risorse sanitarie - pure raccolte nel corso delle sperimentazioni - e relativi costi si potrebbe osservare che, al fine di essere veramente utile ai responsabili decisionali di un dato Paese, un' analisi farmacoeconomica dovrebbe rifarsi a modalità di assistenza reali nel contesto dei

\begin{tabular}{|lcccc|}
\hline Costi (euro) & Caso base & 1) $>$ mortalità & 2) anni scontati & 3) $>$ dose AT III \\
\hline /pzt sopravv. & 119.329 & 137.381 & 119.324 & 70.433 \\
\hline /anno vita guad. & 9.660 & 13.436 & 12.284 & 5.702 \\
\hline /QALY & 13.999 & 19.472 & 17.803 & 8.263 \\
\hline
\end{tabular}

Tabella 2

Parametri di costo/ efficacia 
responsabili stessi; ciò va inteso a due livelli:

- tali modalità dovrebbero essere quelle tipiche delle normali situazioni cliniche, non quelle - più o meno particolari, teoriche che si ritrovano nelle sperimentazioni;

- tali modalità dovrebbero essere quelle seguite localmente, non quelle relative ad ambiti stranieri, più o meno lontani nella normativa e nella prassi.

Non è tuttavia sempre possibile disporre di informazioni del genere. Si cerca allora di localizzare l'analisi per lo meno applicando costi unitari che siano specifici del Paese di riferimento.

Tali sono i limiti di impostazione del presente lavoro - peraltro comuni a gran parte della letteratura di valutazione farmacoeconomica.

Ciò premesso, i risultati del modello applicato all'analisi del caso base indicano che, trattando con dotrecogin alfa, il costo per paziente sopravissuto è pari a euro 119.329, il costo per anno di vita guadagnato a euro 9.660, il costo per QALY a euro 13.999.

Per poter esprimere un apprezzamento definitivo sui dati qui presentati rimane però da confrontarli con alcuni valori orientativi, cioè con delle soglie di accettabilità economico- sociale che abbiano già riscosso riscontri e consensi.

Un primo di tali valori può essere quello, spesso richiamato, per cui un intervento sanitario può ritenersi cost effective se il costo per anno di vita guadagnato si colloca attorno a euro 26.000 [15].

Un altro criterio [16] suggerisce di verificare che il costo per anno di vita guadagnato sia inferiore al PIL (Prodotto Interno Lordo) procapite - che, nel caso dell'Italia, può essere calcolato pari a circa euro 20.000 [7].

Entrambi tali valori sono largamente superiori al costo per anno di vita guadagnato che è stato stimato nel caso base del presente lavoro (euro 9.660), come pure agli analoghi parametri ritrovati nella casistica esplorativa dell'analisi di sensibilità.

$\mathrm{Si}$ dovrebbe così poter concludere che dotrecogin alfa rappresenta un'opzione economicamente valida per il trattamento dei pazienti con sepsi grave in Italia.

Questo lavoro è stato reso possibile da un finanziamento messo a disposizione da Eli Lilly S.p.A.

Un ringraziamento particolare a Derek Angus, the Prowess Economic Team (clo Ely Lilly Indianapolis).

\section{BIBLIOGRAFIA}

1. Parrillo JE, Parker MM, Natanson C, Suffredini AF, Danner RL, Cunnion RE - Sceptic shock in humans; advances in the understanding of pathogenesis, cardiovascular dysfunction, and therapy - Annals of Internal Medicine, 113, 227-242, 1990

2. Bernard GR, Vincent J-L, Laterre P-F, et al. Efficacy and safety of recombinant human activated protein C for severe sepsis. NEJM, 344 (10), 699-709, 2001

3. Drabinski A, Williams G, Formica C. Observational Evaluation of Health State Utilities among a Cohort of Sepsis Patients. Value in Health, (4) 2:128,2001

4. Draugalis JR, Bootman JL, Larson LN, McGhan WF. Pharmacoeconomics: Current Concepts. Edizione italiana a cura di C. Lucioni, Centro per l'Informazione Sanitaria, Milano, 1991

5. Messori A, Vacca F, Vaiani M, Trippoli S and the "Gruppo di Studio sull'antitrombina III". Antithrombin III in patients admitted to intensive care units: a multicenter observational study. Submitted to Critical Care

6. Expert panel. Studio pitre, Milano, non pubblicato

7. ISTAT. Annuario Statistico Italiano. Istituto Nazionale di Statistica, Roma, 2001

8. Quartin AA, Schein RMH, Kett DH, et al. Magnitude and duration of the effect of sepsis on survival. JAMA, 277, 1058-1063, 1997 
9. Brooks R with the EuroQol Group. EuroQol: the current state of play. Health Policy, 37, 53-72, 1996

10. Drabinski A, Williams G, Formica C. Observational Evaluation of Health State Utilities among a Cohort of Sepsis Patients. Value in Health, (4) 2:128,2001

11. Cavallo MC, Lazzaro C, Tabacchi M, Langer M, Salvo I, Serra G, Taddei C. Il costo del reparto di terapia intensiva in Italia: risultati da un'indagine empirica su un campione di 12 Centri. Minerva Anestesiologica, 67, 41-53, 2001

12. Lucioni C, Currado I, Langer M, Mazzi S. I costi della sepsi in Italia. Farmeconomia e percorsi terapeutici, 2 (3), 139148,2001

13. L'Informatore Farmaceutico. OEMF, Milano, 2002

14. Baudo F, Caimi TM, de Cataldo F, Ravizza A, Arlati S, Casella G, Carugo D, Palareti G, Legnani C, Ridolfi L, Rossi R, D’Angelo A, Crippa L, Giudici D, Gallioli G, Wolfler A, Calori G. Antithrombin III (ATIII) replacement therapy in patients with sepsis and/or postsurgical complications: a controlled double-blind, randomized, multicenter study. Intensive Care Med, 24, 336-342, 1998

15. Messori A, Trippoli S. La farmacoeconomia come disciplina scientifica: panoramica delle principali metodologie ed esempi. Giornale di Farmacoeconomia, 2, 176-189, 1998

16. Miller MA, McCann L. Policy analysis of the use of hepatitis B, haemophilus influenzae type B, streptococcus pneumoniae-conjugate and rotavirus vaccines in national immunization schedules. Health Economics, 9, 19-35, 2000

17. Bone RC. Toward an Epidemiology and Natural History of SIRS (Systemic Inflammatory Response Syndrome). JAMA, 268 (24), 3452-3455, 1992 